\title{
Performance Analysis of AODV, CBRP, AOMDV, DSR MANET Routing Protocol using NS2 Simulation
}

\author{
Shalini Kushwaha \\ Department of CSE, \\ IET College \\ Alwar, India
}

\author{
Deepak Chaudhary \\ Department of CSE, \\ IET College, \\ Alwar, India
}

\begin{abstract}
Mobile ad hoc network is a self-directed structure of mobile nodes connected by wireless links. All nodes operate not only as an end system, but also as work as a router to forward the packets. Ad hoc wireless networks are characterized by multihop wireless connectivity, infrastructure less and habitually changing topology. It may be necessary for one mobile node to schedule other hosts for forwarding a packet from source to destination node due to the constrained transmission range of wireless network interfaces. Therefore a self-motivated routing protocol is required for these networks to work properly. A number of Routing protocols have been created to achieve this task. In this paper, a comparative analysis of four reactive routing protocols namely AODV, AOMDV, DSR and CBRP is done. Here CBRP is also a hierarchical routing protocol. This paper is aimed to analyze the adequacy of considered routing protocols in an energy constrained environment under varying mobility and pause time. These protocols have been analyzed extensively for various performance parameters (energy consumption, delay, throughput, Packet Delivery Ratio etc.) over different network scenarios. Simulation results show that none of the protocol surpasses other for all considered scenarios. However, CBRP has produced better results in terms of throughput, normalized routing load and delay while AOMDV is a better choice for energy related parameters. Simulation results of the paper are very helpful for the wise selection of the energy efficient base routing approach to scale MANETs.
\end{abstract}

\section{Keywords}

Routing, Adhoc networks, AODV, AOMDV, DSR, CBRP, Energy

\section{INTRODUCTION}

Over the last decade, researchers have made various researches in the field of mobile computing especially MANETs. A Mobile ad-hoc network (MANETs) is a selforganized, randomly developed network and can easily adopt in working environment. Basically, MANET is the collection of wireless mobile nodes that can interact and communicate with each other, without having the centralized and established infrastructure. MANETs have converted the dream of getting connected "anywhere at any time" in to the reality. MANETs are useful in various application areas such as: communication in the battlefields, institutions and colleges, military areas, disaster recovery areas, law and order maintenance, traffic control areas, medical field, conferences and convocations etc. In MANET, all the nodes are mobile nodes and their topology changes rapidly. The Internet Engineering Task Force (IETF) created a MANET working group to deal with the challenges faced during the construction of the MANET routing protocols. These protocols are basically classified in to three basic types such as: reactive (on demand), proactive (table-driven) and hybrid.
One of the basic goals of the mobile ad-hoc network is to establish correct and efficient route between the mobile nodes so that communication between the sender and receiver is effective.

In Proactive (table driven) routing protocols, each node maintain one or more routing table which contain information about every other node in a network. Routing tables are updated by all the nodes in order to maintain a consistent and up to date view of the network. In table driven routing protocol, continuous broadcasting of messages is done in order to establish routes and maintain them. One of the basic advantages of proactive routing protocol is that route from source to destination is easily available without any overhead, as they are independent of traffic profiles. Various proactive routing protocols are: DSDV [1], [2], DBF [3], GSR [4], WRP [5] and ZRP [6]. In reactive (on demand) routing protocol, creation of routes is done when it is required. When some packets are to be send from source to destination, it may invoke the route discovery mechanism to find the path to the destination. The route is valid, till the destination is reached or it is no longer be required in the future. Some of the reactive routing protocols are: DSR [1] [3], AODV [7] [6] and TORA [2]. In hybrid protocol routing, we combine the benefits of both the reactive as well as proactive. Hybrid protocols are basically dependent on the network size for their functionality.

\section{RELATED WORKS}

Mukesh Kumar et al. [8] compared the performance of AODV and DSR, both of them are reactive routing protocols and Cluster Base Routing Protocol (CBRP) which is a hierarchical reactive routing protocol. Results are obtained by running simulations with different scenarios in GLOMOSIM. These routing protocols were compared in terms of (a) Packet delivery (b) routing overhead and (c) Average delay when subjected to change in pause time and varying no. of nodes. After the comparison of the routing protocol with the help of simulation result they concluded that cluster structure bring scalability and routing efficiency for a MANET as the network traffic load or network size increases. A more stable cluster structure brings efficiency in route discovery and maintenance whereas a less overlapping cluster structure brings efficiency in routing overheads reduction S.R. Biradar et al. [9] compared and evaluated the performance of two type of on demand routing protocol namely Adhoc on demand routing protocol(AODV) which is unipath and Adhoc on demand multipath distance vector (AOMDV) routing protocol . NS-2 simulator is used to check the performance of AODV and DSR. The protocol maintain a send buffer of 64 packet .It contain all data packet waiting for route such as packet waiting in a buffer for more than 30seconds are dropped. A flat topology of $1000 \mathrm{~m} \times 1000 \mathrm{~m}$ is considered for 50 nodes for a simulated time of 100 seconds. The performance metric evaluated are (a) packet delivery (b) Average delay (c) 
Routing overhead (d) No of packet dropped. In simulation, node movement is due to the random way point model. The scenario file used for each simulation is characterized by different pause time. Paper victimizes that AOMDV is better than AODV. AOMDV incurs more routing overhead. It is also efficient in terms of successful packet delivery. Hence, authors concluded that when the network load tolerance is not considered exclusively AOMDV is better on demand routing protocol than AODV. Since it provide better packet delivery and less no of packet dropped .But it increases high overhead than AODV .Mohammed A. Mahdi et al [10] have analyzed the performance of CBRP, AODV and DSR in dense and sparse topology. They realized that CBRP protocol is better than AODV and DSR under dense topology with better packet delivery and less routing load when traffic sources are more than 20. AODV has less delay than CBRP and DSR. In presence of sparse topology, CBRP reacts well than AODV and DSR in term of Routing Load and AODV is better in terms of Packet delivery when sources are (30 and 40). AODV is good even in term of Delay all considered traffic load. With an increase in node's speed in both dense and sparse topology, performance of all protocols degrades. Paper suggests that protocols can also be checked for non-uniform node density.Priyanka Sarkar and Hrituparna [11], analyzed and compared the performance of TORA, AODV, DSR and DYMO routing protocols. They made comparison of these routing protocol based on the performance metrics like (a) packet delivery (b) delay (c) throughput. At last they concluded that AOMDV performance is better than other protocol in different network scenario. Shivlal Mewada et al. [12] discussed the performance of reactive (DSR and AODV) routing protocols. The authors had described that DSR uses the proactive table-driven routing strategy whereas AODV uses the reactive on demand routing strategy with different routing mechanisms. AODV apply the sequence numbers and have only one route to a destination in its routing table whereas DSR make use of source routing and caching policy to maintain multiple routes per destination. They also mentioned that with an increase in number of nodes for a fixed area of $500 \mathrm{~m} \times 500 \mathrm{~m}$ illustrates that even if the terrain area of the network scenario is kept constant, the behaviour of these routing protocols AODV and DSR changes . They analyzed and mentioned the overall performance of DSR routing protocol for performance matrices, Packet Delivery Fraction as well as throughput is better than that of AODV routing protocols.

\section{DESCRIPTIONS OF STUDIED ROUTING PROTOCOLS}

AODV, DSR, CBRP and AOMDV are the routing protocols that have been analyzed on the anvils of energy parameter. The following section discusses the working of these protocols.

\subsection{Adhoc on demand distance vector routing (AODV)}

The Ad Hoc On - demand Distance Vector Routing protocol is a reactive unicast routing protocol for mobile ad hoc networks [13].As a reactive routing protocol [14], AODV only needs to maintain the routing information about the active paths. In AODV, the routing information is maintained in the routing tables at all the nodes. Every mobile node keeps a next hop routing table, which contains the destinations to which it currently has a route. A routing table entry expires if it has not been used for a pre specific finishing time.
When the communication is taking place between the two nodes in the network they exchange the routing information and keep the information up to date as long the information lasts. When a node wants to send a packet to the destination node it starts the route discovery process in order to establish the route between the source and destination node. Therefore source node sends a route request message to its neighbor node (RREQ).The neighboring node receive the route request and increase the hop count by 1 and send to its neighbor so that RREQ can actually be broadcast. The RREQ message will eventually reach to the destination nodes and the destination node will send the route reply message (RREP). The RREP [15] is sent as a unicast, using the path towards the source node established by the RREQ. Similarly to what happens with RREQs, the RREP message allows intermediate nodes to learn a route toward the destination node. Therefore, at the end of the route discovery process, packets can be delivered from the source to the destination node and vice versa. The route will be maintained as long as it vestiges active that is if the data packets are endlessly travelling from source to the destination. As soon as source node stop sending the data packet and become inactive the link will time out and finally be deleted from the intermediate node routing tables.

If the link breaks nodes broadcast a route error message to the source node to inform about the inaccessible destination. After receiving the (RERR), the source node can start again the route discovery by using the sequence number to guarantee the newness of routes.

\subsection{Dynamic source routing (DSR)}

Dynamic source routing [16] is another popular on demand routing protocol, in which routing take place in two phases: route discovery and route maintenance. The key distinguishing features of DSR is the use of source routing .During the first phase that is route discovery the sender node floods the network with route request (RREQ) packets. Each node receiving the RREQ packets ,rebroadcast it, unless it reaches to the destination or it find a route to the destination in its route cache .Such kind of nodes which find either destination or route to the destination will send( RREP) packet to the original source node. The RREP [15] routes itself back to the source by traversing this path backward. The route carried back by the RREP packet is cached at the source for future use. If any link on a source route is broken, the source node is notified using a route error (RERR) packet. The source removes any route using this link from its cache. A new route discovery process must be initiated by the source if this route is still needed. DSR makes very aggressive use of source routing and route caching.

Route maintenance is accomplished through the use of route error packets (RERR) and acknowledgments. The advantage of the protocol is that it uses the route cache information efficiently to reduce the control overheads. The disadvantage of this protocol is that the route maintenance mechanism does not locally repair the broken links.

\subsection{Cluster based Routing Protocol (CBRP)}

Cluster-Based Routing Protocol (CBRP) [18] is a hierarchical on-demand routing protocol that uses source routing, similar to DSR, to avoid forming loops and route packets The goal of clustering is to group the mobile nodes in 'Clusters' in order to organize the nodes in form of a hierarchy, so that significant improvement can be made in the network performance, specifically with large number of nodes. CBRP [16][17] is a pioneer clustering protocol submitted by the mobile Ad Hoc networking working group of the Internet 
Engineering Task Force (IETF). A cluster-based routing scheme usually consists of two parts: Clustering Algorithm and Routing Algorithm.

In CBRP, the protocol divides the nodes of the network into a number of overlapping or disjoint 2-hop-diameter clusters in a distributed manner. Each cluster selects one node as a cluster head which coordinates data transmission within the cluster and with other clusters.. These cluster head node are responsible for the routing process. Other node in a cluster can have a role of cluster gateway or simply a cluster member.

The advantage of CBRP is that only cluster heads exchange routing information, therefore the number of control overhead transmitted through the network is far less than the traditional flooding methods.

As a summary, the CBRP has the following features [18]:

- Fully distributed operation.

- Less flooding traffic during the dynamic route discovery process.

- Explicit exploitation of uni-directional links that would otherwise be unused.

- Broken routes could be repaired locally without rediscovery.

- Sub-optimal routes could be shortened as they are used.

\subsection{Ad hoc On-Demand Multipath Distance Vector Routing}

AOMDV [19] [20] on the other hand is a multi-path routing protocol. It is an extension to AODV and also provides two main services i.e. route discovery and maintenance. Unlike AODV [21], every RREP is being considered by the source node and thus multiple paths discovered in one route discovery. Being the hop-by-hop routing protocol, the intermediate node maintains multiple path entries in their respective routing table. As an optimization measure, by default the difference between primary and an alternate path is equal to 1 hop. The route entry table at each node also contains a list of next hop along with the corresponding hop counts. Every node maintains an advertised hop count for the destination. Advertised hop count defined as the "Maximum hop count for all the paths". Route advertisements of the destination are sent using this hop count. An alternate path to the destination is accepted by a node if the hop count is less than the advertised hop count for the destination.

\section{SIMULATION ENVIRONMENTS}

The Network simulator NS-2 has been used to observe the energy behavior for AODV, DSR, CBRP, AOMDV protocols with respect to the mobility of the nodes and traffic load on the network. Different Simulation parameters which are being used for a 100 node network over AODV, DSR, CBRP AND AOMDV protocols are shown in Table 1.

Table 1: Simulation Parameter

\begin{tabular}{|l|l|}
\hline Simulation Area & $1000 \times 1000 \mathrm{~m} 2$ \\
\hline Protocols used & $\begin{array}{l}\text { AODV ,DSR, CBRP and } \\
\text { AOMDV }\end{array}$ \\
\hline $\begin{array}{l}\text { power consumption for } \\
\text { Transmission }\end{array}$ & $1.6 \mathrm{~W}$ \\
\hline
\end{tabular}

\begin{tabular}{|l|l|}
\hline $\begin{array}{l}\text { power consumption for } \\
\text { Reception }\end{array}$ & $1.2 \mathrm{~W}$ \\
\hline Speed of nodes & $1 \mathrm{~m} / \mathrm{sec}$ to $20 \mathrm{~m} / \mathrm{sec}$ \\
\hline Network size & 25 \\
\hline $\begin{array}{l}\text { Energy supplied to each } \\
\text { node }\end{array}$ & 100 joules \\
\hline Mobility Model & RWP \\
\hline Data Rate & $2 \mathrm{Mbps}$ \\
\hline Transmission Range & $250 \mathrm{mtr}$ \\
\hline Traffic Source & CBR \\
\hline Packet size & 512 byte \\
\hline
\end{tabular}

The results of the simulations performed on CBRP, AODV,AOMDV \& DSR, when the network consists of CBR traffic sources are presented in this section. The comparison of these protocols with respect to varying speed of the mobile nodes and for pause time for different performance metrics are discussed and shown below for small network :

\subsection{Total Energy Consumed}

Total energy consumed is the sum of the energy dissipated by all the nodes after each simulation run.

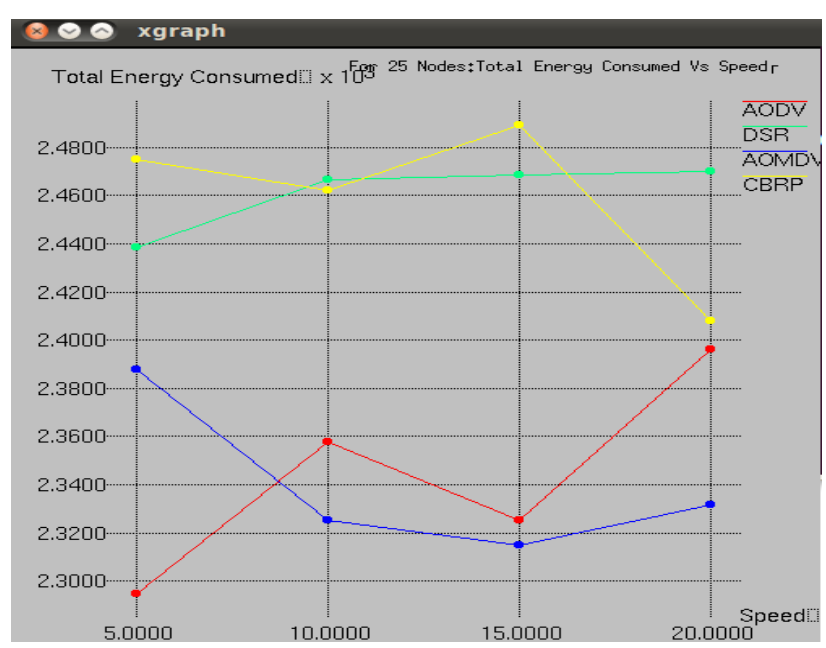

Fig 1: Total Energy Consumption against different speed of nodes

Fig 1 shows that that total energy consumption is high in DSR for the speed 10 to $20 \mathrm{~m} / \mathrm{sec}$. AODV start consuming maximum energy than AOMDV up to $20 \mathrm{~m} / \mathrm{sec}$. CBRP is parallel to DSR at $10 \mathrm{~m} / \mathrm{sec}$ but later it consume less energy at the speed of $20 \mathrm{~m} / \mathrm{sec}$. So it is conclude that AODV consumes very low energy than DSR and CBRP for the speed up to 20 $\mathrm{m} / \mathrm{sec}$ but AOMDV in comparison to AODV is consuming lesser energy as the speed is increases. DSR performance is worst. Thus AOMDV is a better protocol in terms of energy consumption in varying mobility scenario. 


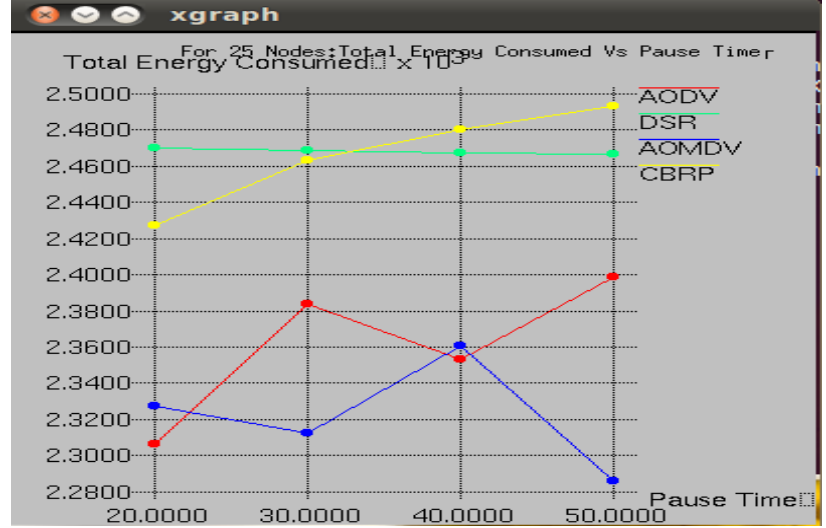

Fig 2: Total energy Consumption against different Pause time

It is clear that Total energy consumption is high in DSR for the pause time 20 to 30 in comparison to AODV, CBRP, and AOMDV. AODV energy consumption is lower at pause time 20 than AOMDV, afterward it start increasing till pause time 30 but again it was reduced at pause time 40 , later it raised a rapidly at reached higher than AOMDV at pause time 50 . CBRP energy consumption was lower than DSR at pause time 20 but it rapidly increased and reached highest at pause time 50 in comparison to AOMDV, DSR, AODV. AOMDV in comparison to AODV is consuming lesser energy as the pause time is increasing. DSR performance is worst. Thus AOMDV is a better protocol in terms of energy consumption in varying pause time.

\subsection{Average Energy Consumed}

Average energy consumed is the energy obtained at each alive node to the number of alive nodes after each simulation run.To evaluate the performance of AODV, DSR, AOMDV and CBRP in term of average energy consumption after each simulation run, different network scenarios have been considered.

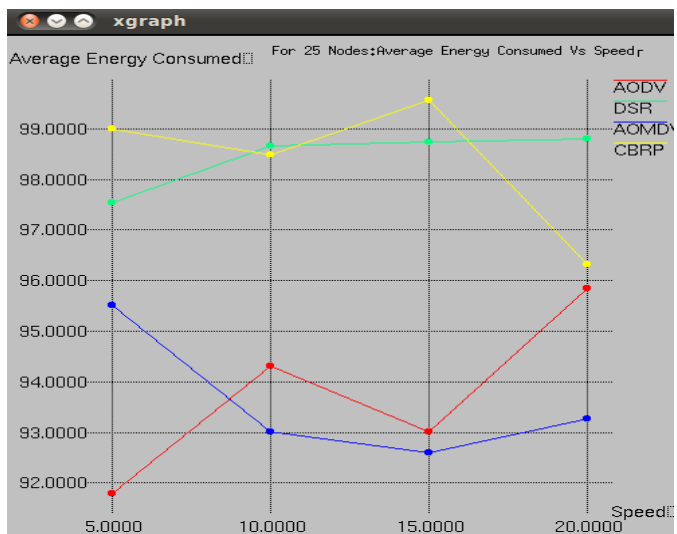

Fig 3: Average Energy Consumed against different speed of nodes

Fig 3 shows that AODV start rising and consume maximum average energy than AOMDV up to $20 \mathrm{~m} / \mathrm{sec}$. CBRP avg energy consumption is higher at speed $5 \mathrm{~m} / \mathrm{sec}$ in comparison to DSR but it was reduced later at speed of 10 and increased again at speed of 15 and later decreased gradually as the speed increase to $20 \mathrm{~m} / \mathrm{sec}$.AOMDV average energy consumption is gradually deceasing from speed 5 to $20 \mathrm{~m} / \mathrm{sec}$ in comparison to AODV, DSR and CBRP .DSR performance is worst. Thus AOMDV is a better protocol in terms of energy consumption in varying mobility scenario.

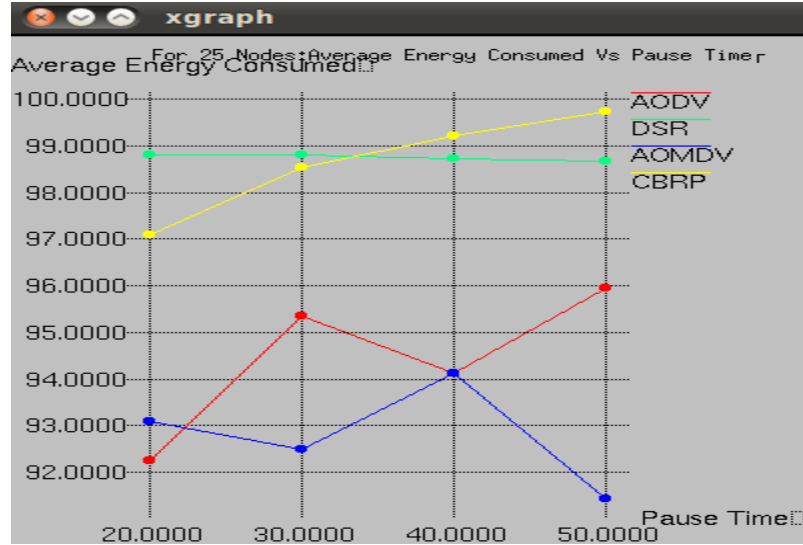

Fig 4: Total energy Consumption against different Pause time

Fig 4 shows that average energy consumption is high in DSR for the pause time 20 to 30 in comparison to AODV, CBRP, and AOMDV. AODV energy consumption is lower at pause time 20 than AOMDV, gradually it start increasing till pause time 30and later became parallel to AOMDV at pause time 40 but gradually it raised again rapidly at reached higher than AOMDV at pause time 50. CBRP energy consumption was lower than DSR at pause time 20 and 30 but it rapidly increase and reached highest at pause time 50 in comparison to AOMDV, DSR, and AODV. AOMDV in comparison to AODV is consuming lesser energy as the pause time is increasing. DSR performance is worst. Thus AOMDV is a better protocol in terms of average energy consumption in varying pause time.

\subsection{Total Delay}

Delay defined as the total latency experienced by a packet to traverse the network from the source to destination. Delay over MANETs has many types such as routing delay, which is the required time to find the path from source to destination. It is calculated as following:

$$
\text { Total Delay }=(\mathrm{Tr}-\mathrm{Ts})
$$

Where $\mathrm{Tr}$ is receive Time and $\mathrm{Ts}$ is sent Time.

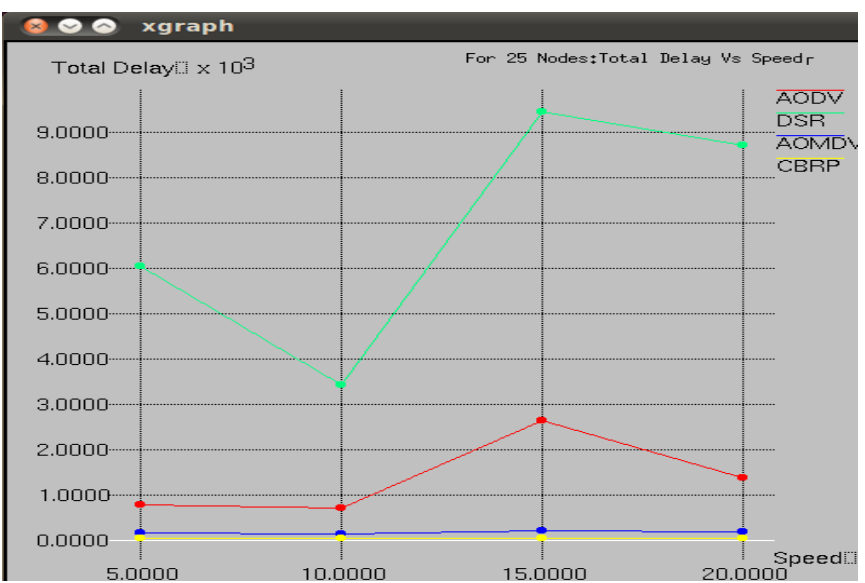

Fig 5: Total Delay against different speed of nodes

Fig 5 shows that DSR total delay is highest .Total delay of CBRP is least in comparison to AODV, AOMDV and DSR. DSR performance is worst. Thus CBRP is a better protocol in terms of Total delay of varying mobility scenario. 


\section{$\otimes \otimes \otimes x g r a p h$}

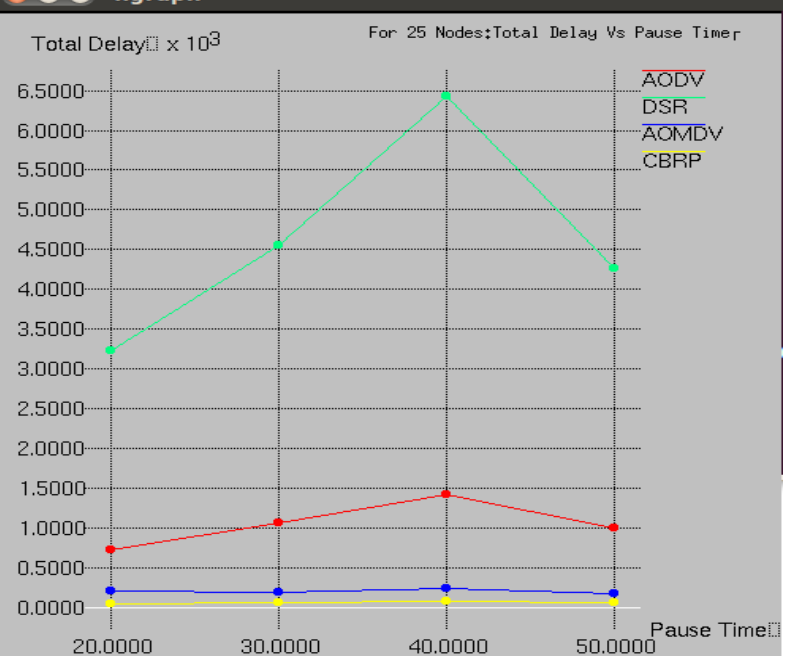

Fig 6: Total Delay against different Pause time

Fig 6 shows that DSR total delay is highest .Total delay of CBRP is least in comparison to AODV, AOMDV and DSR. DSR performance is worst. Thus CBRP is a better protocol in terms of Total delay of varying pause time.

\subsection{Average Delay}

The average end-to-end delay of data packets is the interval between the data packet generation time and the time when the last bit arrives at the destination. It is calculated as following:

$$
\text { Average Delay }=\frac{\sum_{1}^{n} C B R \operatorname{Ren} t_{\text {time }}-C B R \text { receive } r_{\text {time }}}{\sum_{1}^{n} C B R_{\text {Received }}}
$$

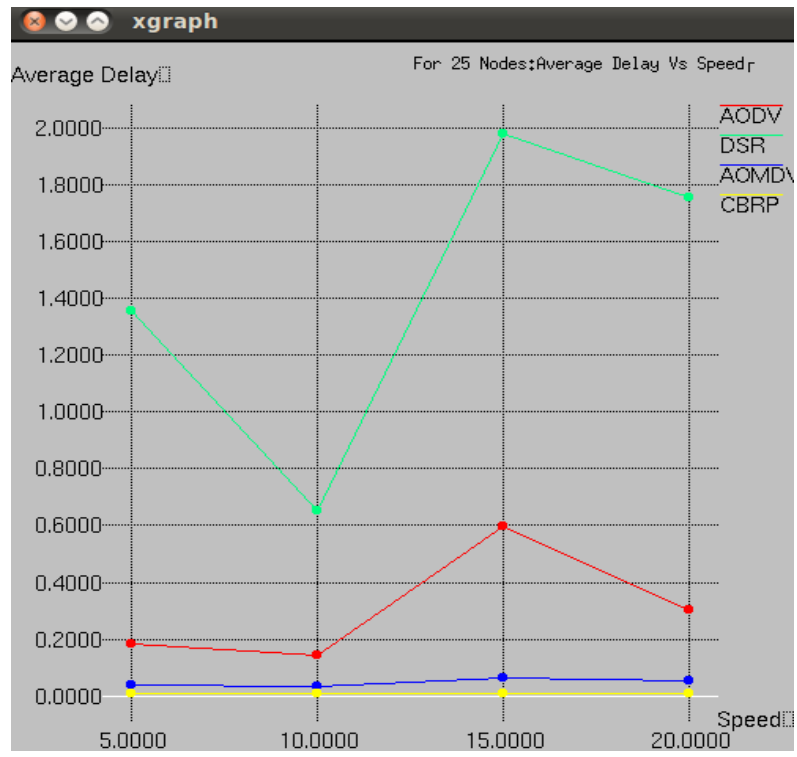

Fig 7: Average Delay against different speed of nodes

Fig 7 shows that Average delay of CBRP is least in comparison to AODV, AOMDV at a speed of 5 to 20 $\mathrm{m} / \mathrm{second}$. Average delay of DSR is highest in comparison to rest of routing protocol at a speed of 5 to $20 \mathrm{~m} / \mathrm{second}$. Hence DSR performance is worst . Thus CBRP is a better protocol in terms of Average delay of varying mobility scenario.

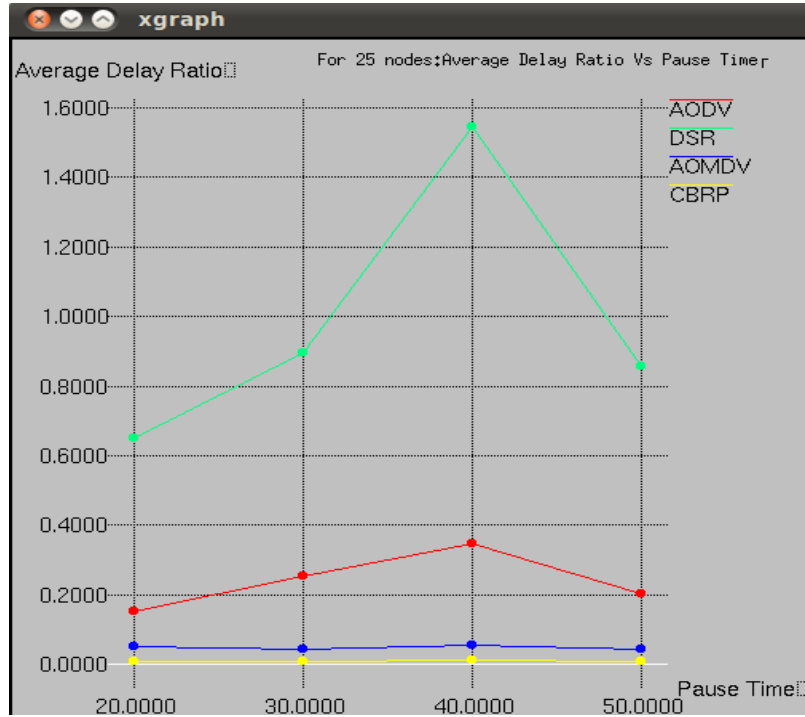

Fig 8: Average Delay against different Pause time

Fig 8 shows that DSR average delay is highest in comparison to AODV, AOMDV and DSR at a pause time of 20 to 50.Total delay of CBRP is least in comparison to AODV, AOMDV and DSR.AODV average delay is higher than AOMDV at a pause time from 20 to 50. DSR performance is worst. Thus CBRP is a better protocol in terms of Average delay of varying pause time.

\subsection{Throughput}

It is defined as the total number of packets delivered over the total simulation time. Mathematically it is defined as:

$$
\text { Throughput }=\frac{\text { Receiver Byte } * 8 /(\text { End time }- \text { Start Time })}{100}
$$

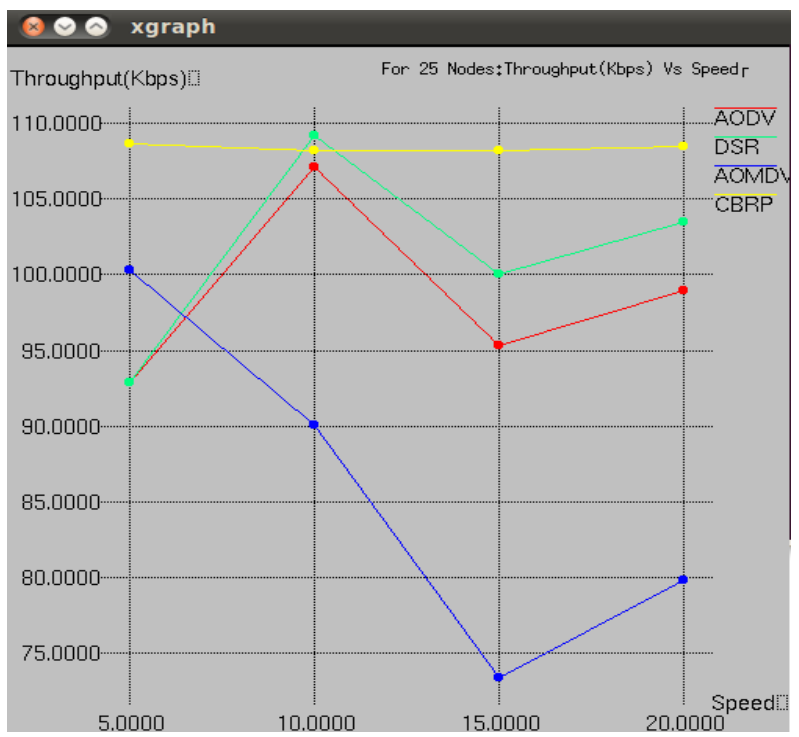

Fig 9: Throughput against different speed of nodes

Fig 9 shows that throughput of CBRP is highest in comparison to AODV, DSR and CBRP.DSR is parallel to AODV at $5 \mathrm{~m} / \mathrm{sec}$ and throughput gradually increases as the speed increases from 10 to $20 \mathrm{~m} / \mathrm{sec}$ in comparison to AODV. AOMDV performance is worst in all, as the speed increasing throughput is decreasing. Thus CBRP is a better protocol in terms of throughput varying mobility scenario. 


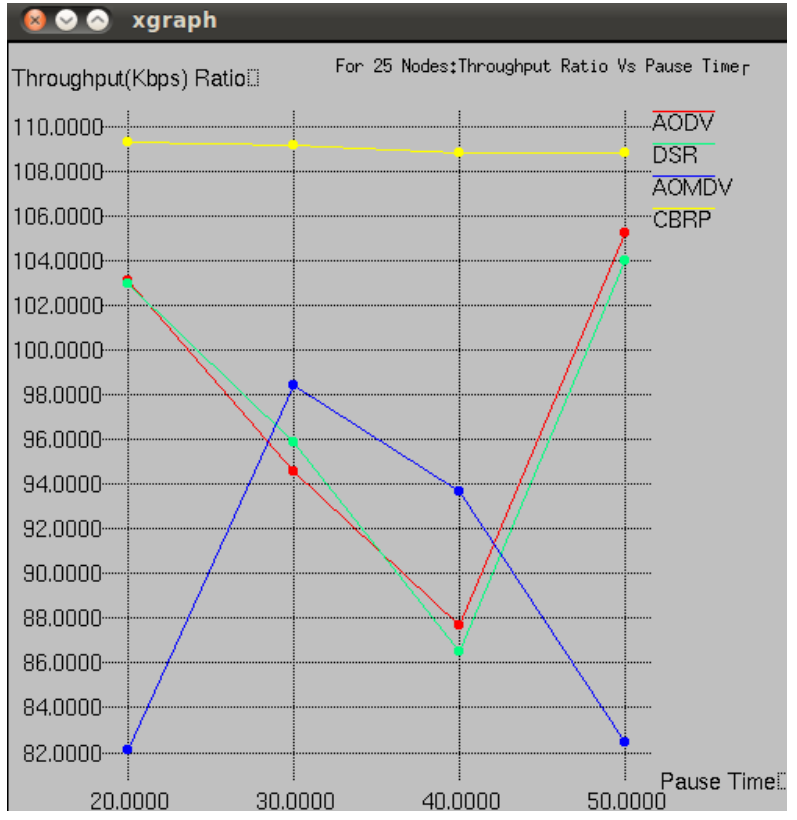

Fig 10: Throughput against different pause time

Fig 10 shows that throughput is very high in case of CBRP. DSR throughput increases at a pause time 20 to 30 and then started gradually decreasing at pause time 40 to 50.AODV and DSR are parallel at 20 pause time and then throughput increases at 30 pause time and it gradually decreases at a pause time 40 to 50 in comparison to AODV. Thus CBRP is a better protocol in terms of throughput with varying pause time.

\subsection{Packet Delivery Ratio}

The ratio of the data packets delivered to the destinations to those generated by the CBR sources.

Packet Delivery Ratio $($ PDR \%) $=$ Number Of Received Packet $* 100$ Number Of Sent Packet

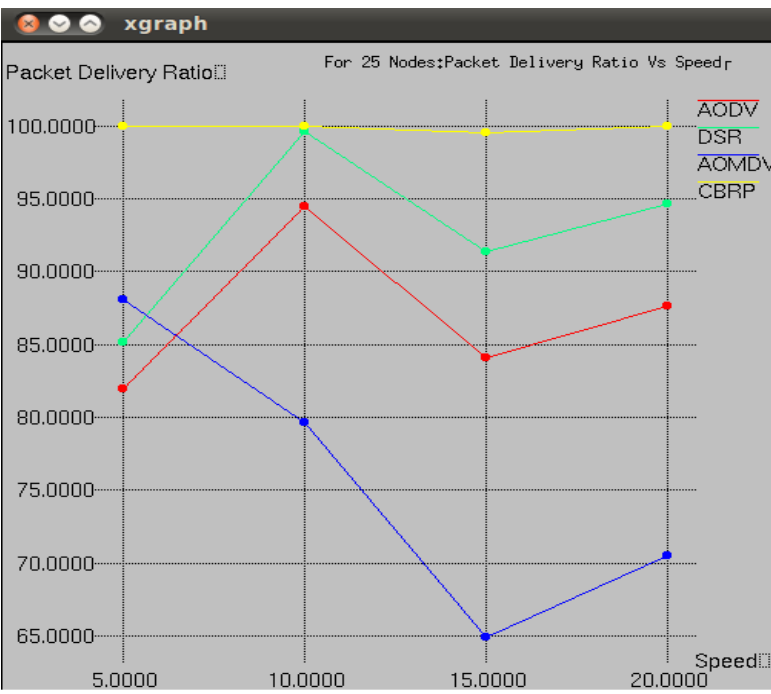

Fig 11: Packet delivery ratio against different speed of nodes

Fig 11 shows that packet delivery ratio is very high in case of CBRP .In AOMDV throughput is very low compare to other. Throughput of DSR is higher than AODV at a speed from 5 to $20 \mathrm{~m} / \mathrm{sec}$. Thus CBRP is a better protocol in terms of throughput with varying speed.

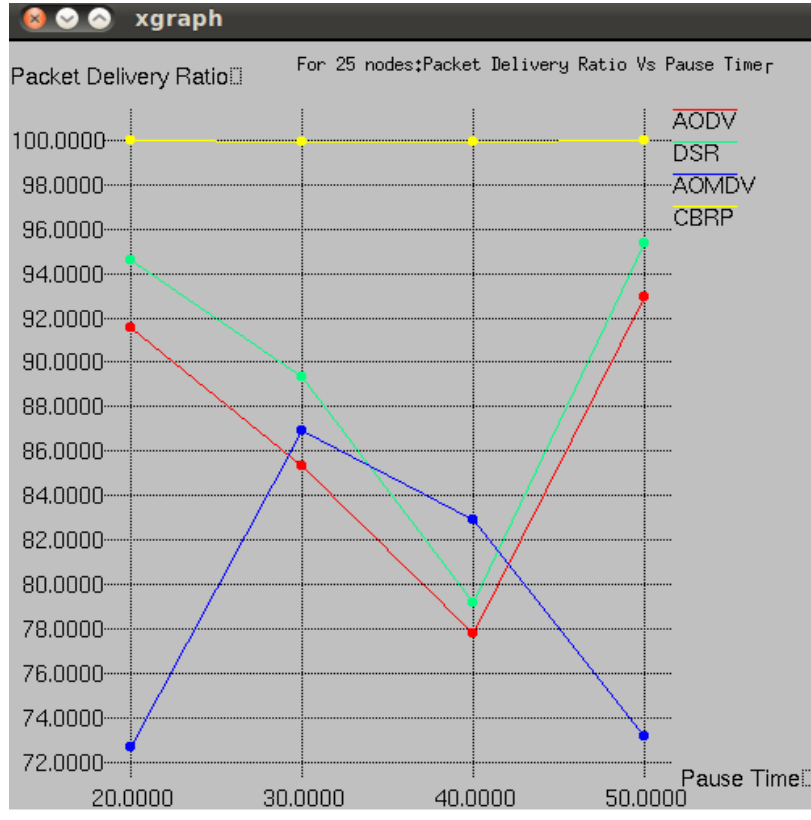

Fig 12: Packet delivery ratio against different pause time

Fig 12 shows that Packet Delivery Ratio of CBRP is highest in comparison to others. Packet Delivery Ratio of AOMDV is lowest in comparison to others. DSR perform well and its packet delivery ratio was higher in comparison to AODV.Thus CBRP is a better protocol in terms of packet delivery ratio with varying pause time.

\section{CONCLUSION AND FUTURE WORKS}

In this paper the energy efficiency of on demand flat MANET routing protocols (AODV, AOMDV,DSR) and hierarchical routing protocols (CBRP) with respect to speed and pause time.

Although none of the considered protocol is a clear winner for all considered network situations but yet the overall performance of CBRP protocols is much better than other three flat routing protocols in term of throughput, packet delivery ratio, average delay and total delay as far as the size of the network is considered. For the additional parameters such as average and total energy consumption by all and alive nodes performance of AOMDV is far much better than other routing protocols. The analytical study of this work is very helpful for the efficient designing of the upcoming MANET routing protocols.In future it can be carried for large network size . This work is still under consideration and it will be continued in future to develop and analyze network scenarios to optimize the tradeoff between capacity and scalability in ad-hoc networks.

\section{REFRENCES}

[1] David B. Johnson and David A. Maltz. Dynamic source routing in ad hoc wireless networks. Technical report, Carnegie Mellon University, 1996.

[2] Mehran Abolhasan, Tadeusz Wysocki, and Eryk Dutkiewicz. A review of routingprotocols for mobile ad hoc networks. Technical report, Telecommunication and Information Research Institute, University of Wollongong, Wollongong, NSW 2522; Motorola Australia Research Centre, 12 Lord St., Botany, NSW 2525, Australia,2003. 
[3] Xiaoyan Hong, Kaixin Xu, and Mario Gerla. Scalable routing protocols for mobile ad hoc networks. 2002.

[4] Integration of mobile ad-hoc networks, EU project DAIDALOS, Susana Sargento, Institute of Telecommunications.

[5] Mobile Ad Hoc Networking: An Essential Technology for Pervasive Computing Jun-Zhao Sun MediaTeam, Machine Vision and Media Processing Unit.

[6] C. Siva Ram Murthy and B. S. Manoj, "Ad Hoc Wireless Networks, Architectures and Protocols", Second Edition, Low price Edition, Pearson Education, 2007.

[7] Jochen Schiller. Mobile Communications. AddisonWesley, 2000.

[8] Mukesh Kumar, Rahul Rishi, D.K Madan. ."Comparative Analysis of CBRP,DSR,AODV routing Protocol in MANET ”, (IJCSE) International Journal on Computer Science and Engineering Vol. 02, No. 09, 2010, pp 2853-2858.

[9] S.R .Biradar, Kaushik Majumder, Subhir Kumar Sarkar. "Performance Evaluation and Comparison of AOD'V and AOMDV", International Journal on Computer Science and Engineering Vol 02 .No.02.

[10] Mohammed A. Mahdi, Tat- Chee Wan "Performance Comparison of Manet Routing Protocol for Dense and Sparse Topology", International Conference on Information and Computer Networks (ICICN 2012) IPCSIT vol. 27 IACSIT Press, Singapore pp 37-41.

[11] Priyanka Sarkar, Hrituparna Paul"Performance Comparison of AODV ,DSR, DYMO and TORA Routing Protocols in Mobile Ad hoc Networks ", International Journal of Recent Trends in Engineering \& Technology, Vol. 11, June 2014.

[12] Shivalal Mewada, Umesh Kumar Singh, Pradeep Kumar Sharma. "Simulation Based Performance Evaluation of Routing Protocols for Mobile Ad hoc Networks (MANET)", IRACST - International Journal of Computer Science and Information Technology \& Security (IJCSITS), ISSN: 2249-9555 Vol. 2, No.4, August 2012.

[13] C.Perkins, "Ad hoc on -demand distance vector (AODV) routing”, RFC 3561, July 2003
[14] Patil V.P, "Reactive and Proactive Routing protocol Energy Efficiency Performance Analysis in Wireless Ad Hoc Networks ", IJCSE) International Journal of Electronics and Computer Science Engineering(IJECSE) ,Vol 1,Number 4,/ ISSN 2277 1956, pp 2333-2341

[15] Kuldeep Singh, "Performance Comparison of Various Routing Protocol with varying number of source nodes", International Journal of Innovations in Engineering and Technology (IJIET) Vol. 1, Vol. 1 Issue 2 August 2012, ISSN: $2319-1058$.

[16] Perkin C. E and Royer E.M "Ad Hoc on Demand Distance Vector Routing ", Proceedings of IEEE Workshops on Mobile Computing Systems and Applications ,pp 90-100.

[17] Awadesh Kumar, Prabhat Singh, Vinay Kumar ,Dr. Neeraj Tyagi, " Performance Analysis of AODV, CBRP, DSDV and DSR MANET Routing Protocol using NS2 Simulation", I. J. Computer Network and Information Security ,pp. 45-50,2013.

[18] Yuvraj Kumbharey, Suwesh Shukla, Sushil Chaturvedi, "Renovated Cluster Based Routing Protocol for MANET", International Journal of Advanced Computer Research Volume-3 Number-1 Issue-8, pp.206-211, 2013

[19] M. Jiang, J. Li and Y.C. Tay, "Cluster Based Routing Protocol (CBRP)", IETF Internet Draft draft-ietf-manetcbrpspec- 01.txt, August 1999.

[20] Mahesh K.Marina and Samir R.Das,"Adhoc Multipath on Demand Distance Vector Routing", Published online in Wiley Inter science, 2006. Pages: 969-988.

[21] Asis Nasipuri , Robert Castarida, Samir R. Das, “ Performance of Multipath Routing for On- Demand Protocols in Mobile Ad Hoc Networks", Mobile Networks and Applications 6, 339-349, 2001 (C) 2001 Kluwer Academic Publishers, Manufactured in The Netherlands.

[22] K Vanaja, DR R Umarani, "An Analysis of Single Path AODV Vs Multipath AOMDV on Link Break Using ns2", International Journal of Electronics and Computer Science Engineering, Volume-1 Number-3,ISSN- 22771956,pp1080-1086. 